\title{
Preliminary assessment of fluorine-18 fluorodeoxyglucose positron emission tomography in patients with bladder cancer
}

\author{
Shigeru Kosuda1, Paul V. Kison'1, Robert Greenough'1, H. Barton Grossman², Richard L. Wahl' \\ 1 Department of Internal Medicine, Division of Nuclear Medicine, The University of Michigan Medical Center, Ann Arbor, Michigan, USA \\ 2 Department of Urology and Cell Biology, The University of Texas M.D. Anderson Cancer Center, Houston, Texas, USA
}

Received 15 December 1996 and in revised form 18 February 1997

\begin{abstract}
The purpose of this study was to assess the feasibility of imaging of bladder cancer with fluorine- 18 fluorodeoxyglucose positron emission tomography (FDG-PET) scanning. We studied 12 patients with histologically proven bladder cancer who had undergone surgical procedures and/or radiotherapy. Retrograde irrigation of the urinary bladder with $1000-3710 \mathrm{ml}$ saline was performed during nine of the studies. Dynamic and static PET images were obtained, and standardized uptake value images were reconstructed. FDG-PET scanning was true-positive in eight patients $(66.7 \%)$, but false-negative in four $(33.3 \%)$. Of 20 organs with tumor mass lesions confirmed pathologically or clinically, 16 $(80 \%)$ were detected by FDG-PET scanning. FDG-PET scanning detected all of 17 distant metastatic lesions and two of three proven regional lymph node metastases. FDG-PET was also capable of differentiating viable recurrent bladder cancer from radiation-induced alterations in two patients. In conclusion, these preliminary data indicate the feasibility of FDG-PET imaging in patients with bladder cancer, although a major remaining pitfall is intense FDG accumulation in the urine.
\end{abstract}

Key words: Bladder cancer - Fluorine-18 fluorodeoxyglucose - Positron emission tomography

Eur J Nucl Med (1997) 24:615-620

\section{Introduction}

Bladder cancer is one of the most common diseases treated by urologists, and is the fourth most common cause of cancer deaths in men after lung, prostate, and colorectal cancer, accounting for $5 \%$ of all cancer deaths in men in the United States [1]. Approximately $70 \%$ of bladder cancers are low-grade, superficial tumors, but the majority of patients develop tumor recurrences fol-

\footnotetext{
* Present address: Department of Radiology, National Defense Medical College, 3-2 Namiki, Tokorozawa 359, Japan

Correspondence to: $\mathrm{S}$. Kosuda
}

lowing endoscopic resection. Most patients with invasive bladder cancer already have invasive disease with occult distant metastases at the time of initial diagnosis [1]. A variety of clinical procedures and many imaging methods are available in the management of bladder cancer. Some of them are routinely performed and are supposed to be useful diagnostic tools for staging bladder cancer. Abdominal ultrasonography, computed tomography (CT), and magnetic resonance imaging (MRI) are helpful in detecting a tumor mass; however, for the purpose of staging they are not as accurate as pathological staging $[1-7]$.

Considerable errors arise when staging bladder cancer with standard diagnostic methods, although accurate staging is very important in planning optimal therapy [ 1 , 2]. For example, the accuracy of CT scanning is limited because it can only detect gross extravesical tumor extension. The presence of enlarged regional lymph nodes does not always indicate metastasis [3]. CT scans fail to detect lymph node metastases of bladder cancer in up to $40 \%$ of patients with such metastases [4]. The accuracy of MRI is only $60 \%$, using TNM staging. Most MRI studies point to the failure of this modality to adequately visualize lymphatic metastases [5]. Transurethral ultrasonography is most valuable in determining the stage of tumor confined to the bladder wall [6]. However, it has limitations in detecting lymph node involvement and perivesical tumor extension. Thus, no single imaging method provides all of the necessary staging information.

Positron emission tomography (PET) with the glucose analogue 2-deoxy-2-[ ${ }^{18}$ F] fluoro-D-glucose (FDG) has been increasingly used as a noninvasive, diagnostic imaging modality for various neoplasms [8-11]. FDGPET can measure tissue metabolism, i.e., increased glycolysis in neoplasm. There have been many published reports regarding FDG tumor metabolic imaging as a diagnostic modality in many diseases. However, no investigators have yet published the accuracy of FDG-PET in patients with bladder cancer, except for one case report which employed FDG-PET to obtain images of preoperative or recurrent bladder cancer $[12,13]$. 
The aim of this preliminary study was to assess the feasibility of imaging of tumors and their regional extension by means of FDG-PET scanning.

\section{Materials and methods}

Patients. We studied 12 patients (seven males, five females) with histologically proven bladder cancer between 1989 and 1994. Their ages ranged from 39 to 83 years, with a mean of 63.5 years. Of the 12 patients, seven had undergone transurethral resection of bladder tumor (TUR-BT), three cystoscopic biopsy, and two radical cystectomy. Four patients had undergone radiotherapy and/or chemotherapy in addition to the surgical procedures. On average the therapies had been performed 2.7 months before FDG-PET study. The histopathologically proven diagnoses were: transitional cell carcinoma in ten cases (nine cases were grade 3, and one, grade 2), adenocarcinoma in one, and high-grade pleomorphic sarcoma in one. All patients had suspected recurrent or residual tumor at the time of the FDG-PET studies. FDG-PET study was thus employed to assess residual or recurrent tumor and its extension, and to differentiate tumor recurrence from radiation-induced cystitis or necrosis. Final diagnoses were obtained by sequential surgical procedures in nine patients: radical cystectomy and ileal conduit in seven patients, emergency laparotomy in one patient with suspected peritoneal carcinomatosis, and aspiration biopsy of a lymph node in one. The duration between the original tissue diagnosis and FDG-PET study was 2 weeks to 7 years, with an average of 15.6 months. In the remaining three patients with distant metastases, the metastatic foci were diagnosed by other diagnostic imaging modalities and review of all available medical records for each patient. All of the patients were followed up after the FDGPET study by the referring urologists and were monitored by chest X-ray, bone scintigraphy, and/or CT.

Written informed consent was obtained from all of the patients after the study was explained to them in detail. The study had Internal Review Board (IRB) approval and was conducted under an Investigational New Drug (IND) accepted by the Food and Drug Administration for FDG. All but one patient fasted for approximately $12 \mathrm{~h}$ before scanning; the remaining patient fasted for $4 \mathrm{~h}$. The serum glucose level was measured before and after scanning, and no patients were found to have high glucose levels or diabetes mellitus. We performed retrograde irrigation of the urinary bladder during the study in nine patients, by placing a double-lumen Foley catheter (12 F), hanging a bag of sterile saline with $1000-3750 \mathrm{ml}$ for irrigation, and then draining the fluid to completely empty the bladder. Postvoid FDG imaging after irrigation was performed in one patient.

Imaging protocol. Fluorine-18 was prepared with a medical cyclotron (T.C.C. model CS-30 cyclotron, Berkeley, Calif.) by the acceleration of deutrons into an $\mathrm{H}_{2}{ }^{18} \mathrm{O}$ target. A nucleophilic fluorination procedure was used to produce FDG of high specific activity (estimated to be greater than $3000 \mathrm{Ci} / \mathrm{mmol}$ ) which was of greater than $95 \%$ radiochemical purity [14].

All patients were studied with a whole-body eight-ring (15 plane) positron emission scanner (Siemens CTI 931 or 921 PET scanner; these scanners had an axial field of view of approximately $10.3 \mathrm{~cm}$ and $15 \mathrm{~cm}$, respectively). The pixel size of the 931 and 921 scanners was 0.469 and 0.422 , respectively. Patients were positioned supine and head first in the scanner gantry with arms above the head so that the arms and head were through the scanner gantry. Transmission scans using a germanium-68 source were performed at the level believed to include the mass lesion. After the transmission images had been obtained, the patient was positioned so that the scanner field (approximately $20-\mathrm{cm}$ field of view) was at the imaging level believed to include the mass; dynamic scan acquisition over 60 min was then performed after intravenous injection of approximately $10 \mathrm{mCi}(370 \mathrm{MBq})$ FDG. A dynamic imaging sequence was used with 10 -s acquisition frames during the initial $1 \mathrm{~min}, 20-\mathrm{s}$ frames between 1 and $2 \mathrm{~min}, 1.5-\mathrm{min}$ frames through $5 \mathrm{~min}$, a single 5 - $\mathrm{min}$ frame through $10 \mathrm{~min}$, and then sequential 10-min image frames through 60 or 70 min after injection. At 60-70 min after injection the patient was repositioned and the second imaging levels were examined for $10 \mathrm{~min}$. In addition, in two patients "whole-body" FDG images were acquired over 15 (three bed positions of 5 min each) or 21 (seven bed positions of $3 \mathrm{~min}$ each) min because of suspected multiple metastases to remote organs.

PET images were reconstructed from projection data, and transaxial, coronal, and sagittal sections were obtained with 15 contiguous slices with a slice thickness of $6.7 \mathrm{~mm}$ and a spatial resolution of $6.1 \mathrm{~mm}$ full-width at half-maximum (FWHM) in the center of the field of view. All data were reconstructed in a $128 \times 128$ image matrix. The final in-plane resolution in reconstructed and Hann-filtered images with a cut-off frequency of 0.3 cycles per pixel was approximately $8 \mathrm{~mm}$ FWHM.

All dynamic and static PET images were taken and interpreted by two nuclear medicine specialists, including sagittal, coronal, and transverse sections. FDG-PET findings were compared with official dictated reports of CT and/or MRI findings, and evaluated.

Tumor accumulation was classified into four grades: grade 0 , normal: no abnormal accumulation; grade 1, probably normal: negative FDG scan; grade 2, probably abnormal: positive FDG scan; grade 3, definitely abnormal: positive FDG scan.

Our visual interpretation criteria for establishing that the activity was from the bladder cancer and not from the urine in the bladder were as follows: correspondence with the lesion on CT and/or MRI, and earlier visualization with gradually increased accumulation on dynamic or influx images.

The standardized uptake value (SUV) was defined as follows:

SUV=decay-corrected dose/cc tumor/injected dose/patient weight $(\mathrm{g})$.

SUV images were reconstructed in 11 patients from projection data obtained at 60-70 min following injection, and SUVs were calculated from regions of interest (ROIs) drawn on sites metabolically representing the most active areas in tumors on images in each patient. The ROIs were usually 16 pixels in size. Tumor accumulation was quantiatively evaluated by the SUVS.

Influx images were generated as follows: An ROI was drawn around the femoral artery to create an input function curve. Then the Patlak parameters were calculated and fitted using the linear least-squares method from 5 to $60 \mathrm{~min}$ post injection for each pixel in the dynamic image sequence. Fits were constrained so the correlation coefficient was 0.9 or greater [15].

\section{Results}

All of the 12 patients were confirmed to have recurrent or residual tumor in the bladder and/or lymph node or distant organ metastasis. Overall, FDG-PET scanning was true-positive (presence of focal FDG uptake relative to surrounding tissue uptake) in eight of the patients $(66.7 \%)$, but false-negative in four $(33.3 \%)$ (Table 1). Of 20 organs with tumor mass lesions confirmed pathologi- 
Table 1. Tumor characteristics and FDG-PET grading for each patient

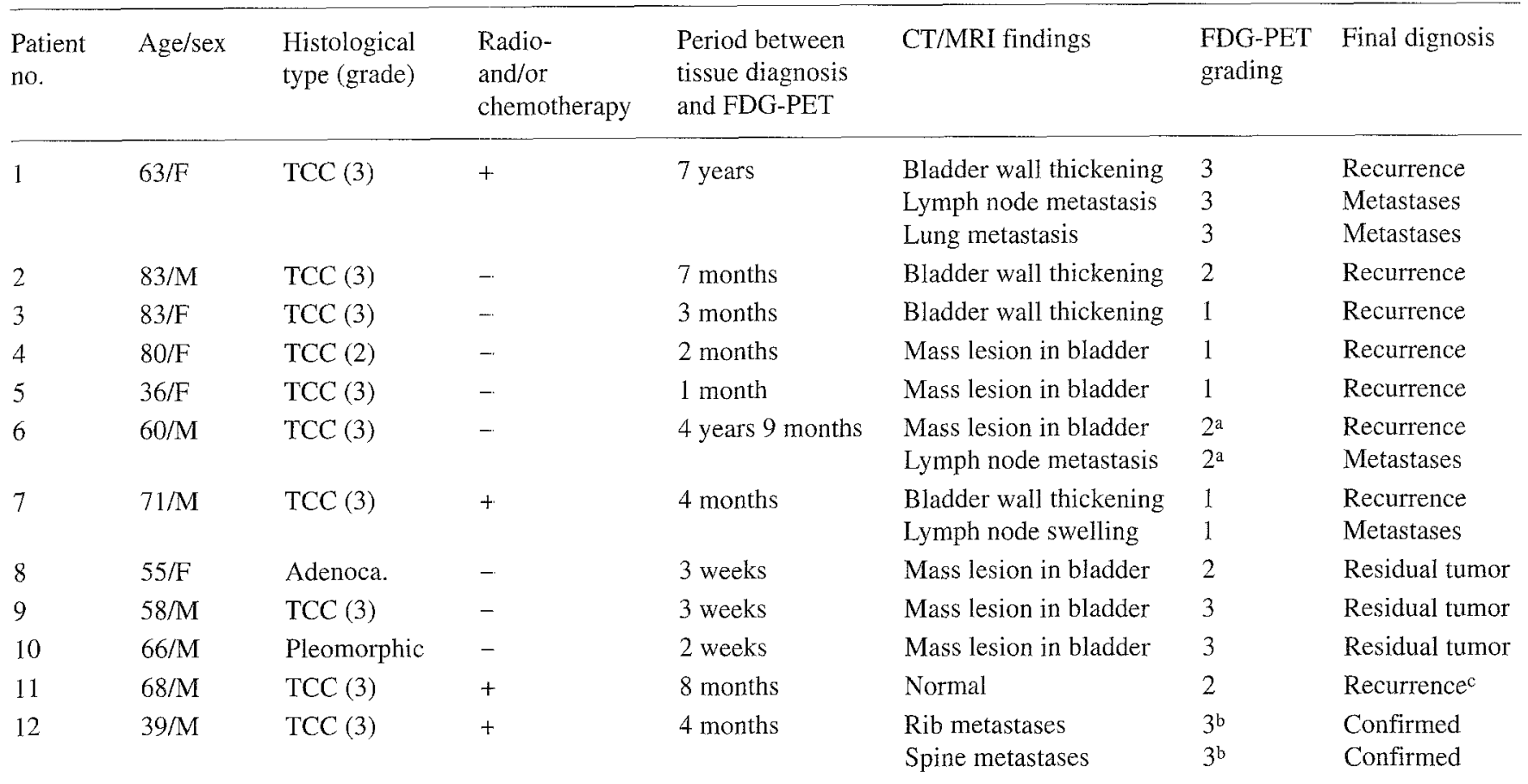

Patients 1-7 had undergone TUR-BT, patients 8-10, cystoscopic biopsy, and patients 11 and 12 , radical cystectomy TCC, Transitional cell carcinoma; Adenoca., adenocarcinoma a Whole-body imaging b Whole-body and PET images revealed metastases of the cerivcal lymph nodes and sternum, as well as rib and spine metastases c Peritoneal carcinomatosis

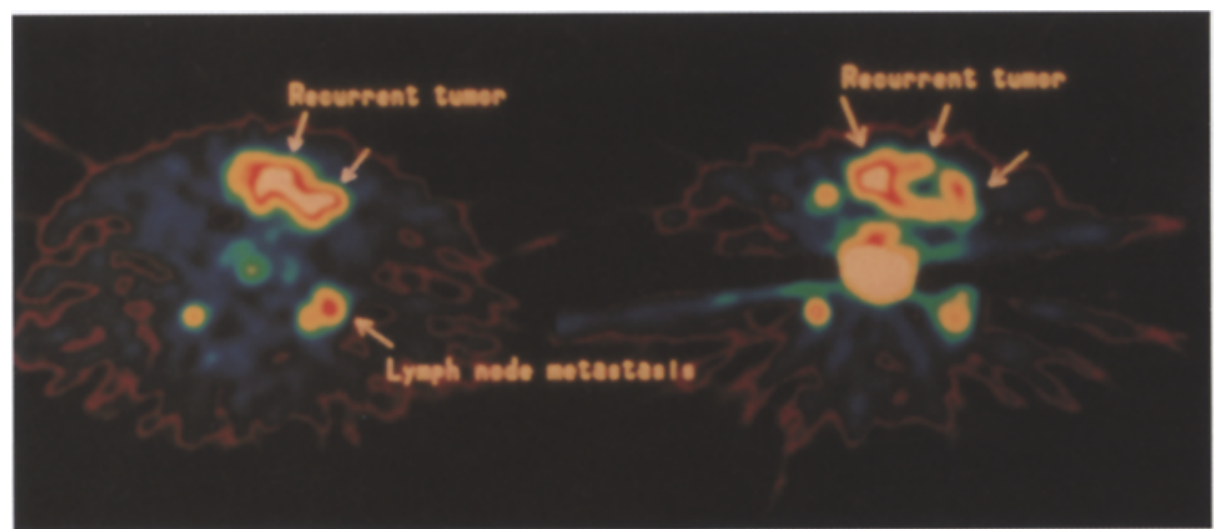

Fig. 1. A transaxial FDG-PET image of the section through the vault of the bladder in a 63-year-old female (patient no. 1) with recurrent cancer who had received radiotherapy and chemotherapy. Recurrent tumor and regional lymph node metastasis (arrow) are well visualized cally or clinically, $16(80 \%)$ were detected by FDG-PET scanning. All of the multiple metastatic lesions ( 17 foci) of distant organs, including the lung (eight foci), bone (eight foci), and a remote lymph node (one focus), were depicted in two patients. In patient no. 12, FDG-PET whole-body scan revealed multiple osseous metastases. FDG-PET scan visualized one intrapelvic mass in patient no. 11 with peritoneal carcinomatosis, who had a normal finding on CT.

Urinary tracer activity was present to some extent in the urinary system (bladder and bilateral ureter) in all of the patients. We were able to decrease the urinary FDG activity by urinary irrigation, but were unable to reduce it to the background level. The internal urinary FDG activity reduced the possibility of detecting lesions in the

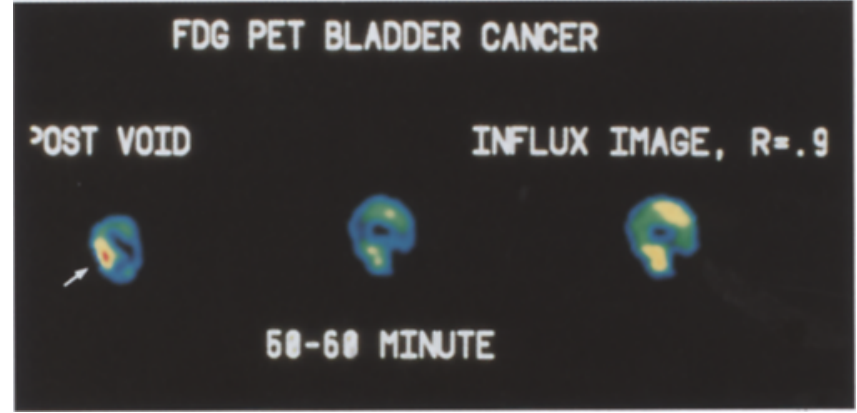

Fig. 2. Postvoid image after irrigation (left), static image 50-60 min following injection (middle), and influx image (right) in a 66year-old male (patient no. 10) with pleomorphic sarcoma in the bladder. The postvoid image reveals most clearly the tumor localization of FDG (arrow) 

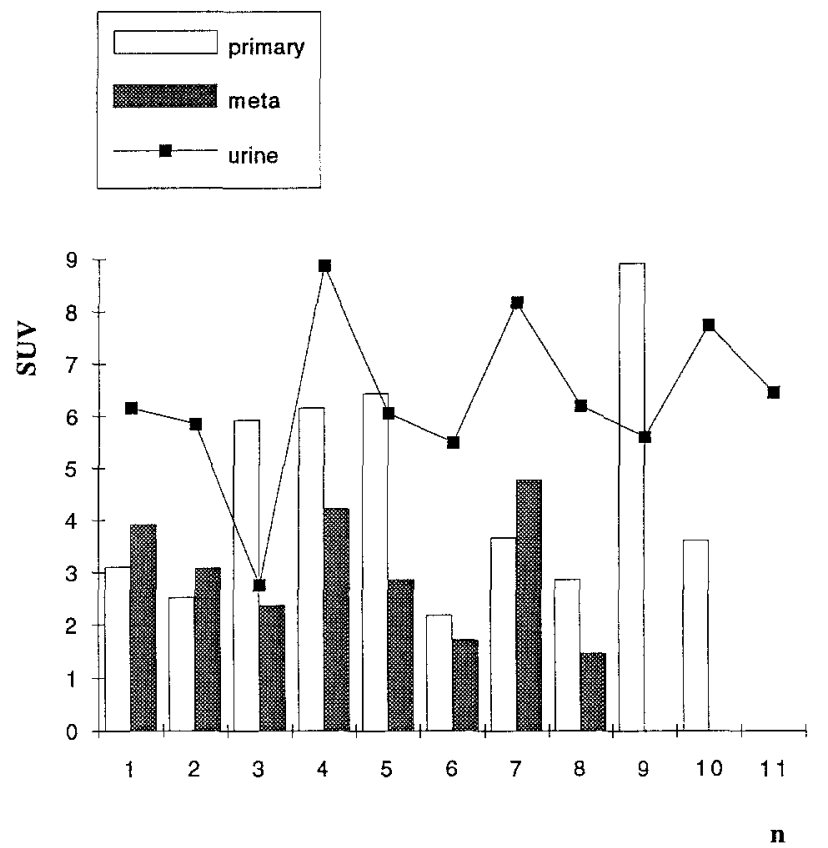

Fig. 3. Distribution of the SUVs of primary $(n=10)$ and metastatic $(n=8)$ lesions of bladder tumors, compared with the SUVs $(n=11)$ of the urine in the bladder and ureters

tivity reduced the possibility of detecting lesions in the urinary bladder and in the vicinity of the ureters.

As for detection of regional lymph node metastasis by FDG-PET scanning, of three nodal lesions (of which two were histologically proven), two were detected by FDGPET. In one patient (no. 8), extravesical extension, which CT scan was unable to detect, was depicted by FDG-PET and proven to be sigmoid colon invasion by pathology.

With regard to detection of recurrent or residual tumor in the urinary bladder, FDG-PET scanning was capable of detecting viable bladder cancer in six of the ten patients $(60 \%)$. FDG-PET scanning was also capable of differentiating viable recurrent bladder cancer from radiation-induced thickening or necrosis of the bladder wall in two patients who received irradiation and chemotherapy (Fig. 1).

However, FDG-PET scanning did not detect recurrence in four patients $(40 \%)$, principally because of intense accumulation of FDG excreted in the urinary bladder. All of the patients showed moderate or intense accumulation of urinary FDG in the urinary bladder, despite saline irrigation of it. Post-void FDG imaging and Patlak graphical analysis proved capable of improving the quality of pelvic FDG-PET images (Fig. 2). Influx images resulted in reduction of urinary FDG activity in two patients.

The calculated SUVs ranged from 2.19 to 8.94 $(4.55 \pm 2.20, n=10)$ in the primary sites, and from 1.47 to $4.79(3.06 \pm 1.19, n=8)$ in organ metastases; in the lymph node metastases the SUV averaged 3.64. The SUVs of the urine in the bladder and ureters ranged from 2.77 to $8.88(6.32 \pm 1.62, n=11)$ (Fig. 3).

\section{Discussion}

It has been recognized that the precise staging of bladder cancer is of crucial importance in determining optimal cancer treatment and predicting prognosis. This is particularly so in patients with bladder cancer, who frequently show a recurrent or persistent course. Basic principles of management require an accurate understanding of the location, extent, and viability of the disease during and after treatment as well as the staging. However, as mentioned above, abdominal ultrasonography, CT, and MRI, which are used in the management of bladder cancer, are not as accurate in staging as is pathological staging [1-7]. CT appears to be most widely used in the assessment of bladder carcinoma, but the accuracy of CT findings in respect of perivesical tumor growth or extension to contiguous organs is only $64 \%-92 \%$ [3]. When using CT in the staging of bladder cancer, overstaging is more commonly observed than understaging. The reported accuracy of CT in detecting lymph node involvement ranges from $70 \%$ to $90 \%$, with false-negative rates of $25 \%-40 \%$ [4]. Dynamic contrast-enhanced MRI is more accurate than conventional MRI, although its staging accuracy is at best $75 \%$, with the most common error being overstaging [16]. Barentsz et al. reported that a three-dimensional technique using $\mathrm{T} 1$-weighted magnetization prepared-rapid gradient-echo resulted in a $15 \%$ improvement in staging [17]. However, these findings are only based on structural or anatomical alterations, and they do not reflect alteration in the metabolism of tumor tissues. Generally speaking, these modalities have poor capability for differentiating viable tumor mass from radiation-induced cystitis or radiation necrosis, although such differentiation is important in cases of bladder cancer with a persistent, protracted clinical course. After radiotherapy, the discordance between clinical and pathological staging may approach $50 \%$ due to both under- and overstaging [4].

On the other hand, it is conceivable that alteration in metabolism precedes morphological alteration. FDG, a glucose analog, has been extensively used as a tumorscanning agent in PET metabolic studies. There is, however, little clinical information regarding the utility of FDG-PET in bladder cancer.

In our study we investigated the feasibility of performing FDG-PET in patients with bladder cancer. The detection rate achieved with FDG-PET scanning was $66.7 \%$ ( 8 of 12 patients with bladder tumors), although four intravesical recurrent tumors could not be detected. Of 20 organs with tumor mass lesions confirmed pathologically or clinically, $16(80 \%)$ were detected by FDGPET scanning. FDG-PET scanning depicted two of three regional lymph node metastases in three patients, and whole-body FDG imaging and FDG-PET scanning clearly revealed all of 17 multiple metastatic lesions in two patients. In one case direct invasion of the sigmoid colon, which could not be detected by CT scan, was depicted by FDG-PET. These results suggest that it is feasible to use FDG-PET for clinical evaluation in patients 
with bladder cancer, especially at advanced stages. From the viewpoint of potential cost-effectiveness, FDG-PET might be employed mainly in preoperative patients with probably positive findings on CT and/or MRI, since these modalities have a tendency toward overstanding $[1-5,7,16,17]$.

FDG-PET was very useful in detecting recurrent bladder cancer in two patients who had undergone radiotherapy and chemotherapy, because CT and MRI were unable to differentiate radiation necrosis from recurrent tumor. CT, which currently is the most commonly used diagnostic modality in bladder cancer, has a poor ability to discriminate recurrent cancer from certain benign diseases, i.e., radiation-induced bladder wall thickening or necrosis, and infectious cystitis with hematoma resulting from operative procedures. State-of-the-art MRI equipment also has difficulty in differentiating between radiation necrosis and tumor recurrence. The increase in FDG accumulation, if any, is well correlated with the number of viable cancer cells [18]. Given the high frequency of recurrence of bladder cancer after treatment, there is a possible role for FDG-PET in monitoring patients following treatment. We believe that FDG-PET represents the examination of choice in patients with a mass lesion on CT or MRI after radiotherapy and/or chemotherapy, although our clinical experience is limited.

A major problem with FDG-PET in imaging bladder cancer is the comparatively intense FDG accumulation due to excretion of FDG in the urine [19]. Urinary excretion of FDG in dogs was $16 \%$ of the injected dose at 60 min, and kidneys and urine have the highest FDG uptake in the body, as measured in \% injected dose/organ [20]. We performed retrograde irrigation of the urinary bladder using saline irrigant and a Foley catheter before taking PET images, but urinary activity overlying tumor activity is more or less inevitable on tomographic sections. One of the reasons why four patients with intravesical recurrent or residual tumor in our series had false-negative FDG studies was probably the effect of urinary FDG activity. Thus, FDG-PET would not appear to have a strong likelihood of detecting bladder cancer of stages 0 to $\mathrm{C}$ in the staging system proposed by Jewett-StrongMarshall. However, stage D1, in which there is involvement of the contiguous organs and lymph nodes, might be adequately diagnosed by FDG-PET, as shown in patient no. 1 (Fig. 1). It should be noted that urinary accumulation in the ureters may interfere with regional lymph node accumulation of FDG.

Unfortunately, the SUVs calculated showed no differences between tumor tissue and urine, and thus proved of little additional value, although the SUVs of the urine did show a tendency to be greater than 5.0.

Although urinary activity degraded FDG-PET image quality, influx images in two patients and a postvoid image in one patient reduced the apparent activity of FDG excretion in the urine. Thus, influx or postvoid images after irrigation appear promising techniques for improving lesion detectability, in addition to saline irrigation.
Another method of lowering or diluting FDG activity in the urine, used by many other groups, is the preparation of an intravenous drip infusion of saline and/or diuretics prior to FDG administration.

In summary, these preliminary data further indicate the feasibility of FDG-PET imaging of bladder cancer, although our study population was limited and did not include any patients who were true-negative for bladder cancer lesions. FDG-PET might be useful in detecting perivesical tumor growth or distant metastasis in patients with advanced bladder cancer, and for the early detection of recurrent cancer following therapy, although a major remaining pitfall is the intense FDG accumulation due to excretion in the urine. Further prospective detailed studies of the FDG-PET method in patients with bladder tumors appear warranted, possibly including methods to reduce the urinary activity of FDG.

\section{References}

1. Catalona WJ. Urothelial tumors of the urinary tract. In: Walsh PC, Retik AB, Stamey TA, Darracott Vaughan E, eds. Campbell's urology, 6th edn, vol 2. Philadelphia: Saunders; 1992: 1094-1158.

2. McCarthy $P$, Ramchandani P, Pollack $H$. The bladder and urethra. In: Vanel D, Stark D, eds. Imaging strategies in oncology. London: Martin Dunitz; 1993: 289-297.

3. Nurmi M, Katevuo K, Puntala P. Reliability of CT in preoperative evaluation of bladder carcinoma. Scand J Urol Nephrol 1988; 22: 125-128.

4. Lantz EJ, Hattery RR. Diagnostic imaging of urothelial cancer. Urol Clin North Am 1984; 11: 567-583.

5. Buy J-N, Moss A, Guinet C, et al. MR staging of bladder carcinoma: correlation with pathologic findings. Radiology 1988; 169: 695-700.

6. Koraitim M, Kamal B, Metwalli N, Zaky Y. Transurethral ultrasonographic assessment of bladder carcinoma: its value and limitation. J. Urol 1995; 154: 375-378.

7. Schmidt JD, Weinstein SH. Pitfalls in clinical staging of bladder tumors. Urol Clin North Am 1976; 3: 107-127.

8. Wahl RL. Positron emission tomography: application in oncology. In: Murray IPC, Ell PJ, eds. Nuclear medicine in clinical diagnosis and treatment. Edinburgh: Churchill Livingstone; 1994: 801-882.

9. Rodriguez M, Rehn S, Ahlstrom H, Sundstrom C, Glimelius B. Predicting malignancy grade with PET in non-Hodgkin's disease. J Nucl Med 1995; 36: 1790-1796.

10. Nolop KB, Rhodes CG, Brudin LH, et al. Glucose utilization in vivo by human pulmonary neoplasms. Cancer 1987; 60: 2682-2689.

11. Wahl RL, Cody RL, Hutchins GD, Kuhl DE. Primary and metastatic breast carcinoma: initial clinical evaluation with PET with the radiolabeled glucose analogue 2-[F-18]-fluoro2-deoxy-D-glucose. Radiology 1991; 179: 765-770.

12. Harney JV, Wahl RL, Liebert M, et al. Uptake of 2-deoxy, 2(18F)fluoro-D-glucose in bladder cancer: animal localization and initial patient positron emission tomography. J Urol 1991; 145: 279-283.

13. Hoh CK, Hawkins RA, Glaspy JA, et al. Cancer detection with whole-body PET using 2-[ ${ }^{18}$ F]fluoro-2-deoxy-D-glucose. $J$ Comput Assist Tomogr 1993; 17: 582-589. 
14. Toorongian SA, Mulholland GK, Jewett DM, Bachelor MA, Kilbourn MR. Routine production of 2-deoxy-2-[18F]fluoro-Dglucose by direct nucleophilic exchange on a quarternary 4 aminopyridinium resin. Int $J$ Rad Appl Instrum 1990; 17: 273-279.

15. Zasadney KR, Wahl RL. Enhanced FDG-PET tumor imaging with correlation-coefficient filtered influx-constant images. $J$ Nucl Med 1996; 37: 371-374.

16. Kim B, Semelka RC, Aschner SM, Chalpin DB, Carroll PR, Hricak H. Bladder tumor staging: comparison of contrast-enhanced CT, T1- and T2-weighted MR imaging, dynamic gadolinium-enhanced imaging, and late gadolinium-enhanced imaging. Radiology 1994; 193: 239-245.

17. Barentsz JO, Jager G, Mugler III JP, et al. Staging urinary bladder cancer: value of T1-weighted three-dimensional mag- netization prepared-rapid gradient-echo and two-dimensional spin-echo sequences. AJR 1995; 164: 109-115.

18. Higashi K, Clavo AC, Wahl RL. Does FDG uptake measure proliferative activity of human cancer cells? In vitro comparison with DNA flow cytometry and tritiated thymidine uptake. J Nucl Med 1993; 34: 414-419.

19. Effert PJ, Bares R, Handt S, Wolff JM, Bull U, Jakse G. Metabolic imaging of untreated prostate cancer by positron tomography with ${ }^{18}$ fluorine-labeled deoxyglucose. J Urol 1996; 155 : 994-998.

20. Gallagher BM, Ansari A, Atkins H, et al. Radiopharmaceuticals XXVII. ${ }^{18} \mathrm{~F}$-labeled 2-deoxy-2-fluoro-D-glucose as a radiopharmaceutical for measuring regional myocardial glucose metabolism in vivo: tissue distribution and imaging studies in animals. J Nucl Med 1977; 18: 990-996. 